\title{
Efecto de tomar un curso en línea sobre el aprendizaje de los estudiantes de agroindustria en la Universidad Estatal a Distancia de Costa Rica
}

\author{
Carmen Andrés Jiménez \\ Universidad Estatal a Distancia, 474-2050 Mercedes de Montes de Oca, San José-Costa Rica; candres@uned.ac.cr
}

Recibido 30-XI-2009 Corregido 7-I-2010 Aceptado 1-II-2010

\begin{abstract}
Effect of taking an online Agroindustry course on student learning at the Costa Rican Distance Education University. Learning online is a challenge that is becoming common around the world, for study and work. The Costa Rican Distance Education University is motivating students to learn through this system. Agroindustry was the first course in the agronomy career that was taught online. To study the effect of the online modality on learning, the course contents were adapted for online teaching and the students performance and opinion about the course were recorded. The evaluation and learning were positive: Agroindustry can be successfully taught in this university using online materials.
\end{abstract}

\section{KEY WORDS}

Agro-industry, virtual, traditional, performance, acceptance, motivation, learning evaluation.

\section{RESUMEN}

El aprendizaje en línea es cada vez más común en todo el mundo y es una herramienta que permite estudiar y trabajar. La Universidad Estatal a Distancia motiva a los estudiantes a aprender a través de este sistema. Agroindustria fue el primer curso en línea que se impartió en la carrera de agronomía. Los contenidos del curso fueron adaptados a esta modalidad. Se estudió el efecto en el aprendizaje al utilizar este método de enseñanza, el rendimiento y la opinión de los estudiantes. La evaluación y el aprendizaje fueron positivos, así que el curso de agroindustria y la utilización de materiales en línea fueron exitosos en esta universidad.

\section{PALABRAS CLAVE}

Agroindustria, virtual, tradicional, rendimiento, aceptación, motivación, evaluación de los aprendizajes.
En casi todo el mundo se están implementando cursos en línea, tanto a nivel educativo como a nivel laboral (Barrón 2004, Miranda 2007, Fonseca 2009). Esta modalidad educativa requiere de un esfuerzo diferente al que estamos acostumbrados porque atiende múltiples dimensiones; desde lo meramente administrativo o logístico hasta el diseño curricular, el diseño de los contenidos o materiales y el diseño de los entornos (Salas 2008).

En la UNED se ha insistido mucho en la necesidad de ampliar la variedad de los materiales de aprendizaje, incluyendo más cursos que utilicen nuevas tecnologías, ya que los egresados encontrarán esto en su campo laboral (Méndez \& Monge 2007). Además que los que ya laboran, encuentran más cómodo y enriquecedor, el llevar un curso en línea que les dé todas las herramientas necesarias sin necesidad de transportarse.
La UNED está dirigiéndose hacia la modalidad virtual, sin dejar de lado la presencialidad en sus videoconferencias, sus teleclases y sus laboratorios. Pero incluso a nivel práctico, se han implementado algunos laboratorios virtuales. En el estudio realizado por Méndez et al. (2002), al realizar la comparación entre los grupos que hicieron los laboratorios reales y los que usaron laboratorios virtuales, se vio que no había diferencias estadísticamente significativas en la tasa de estudiantes que aprobaron el curso, a pesar de que ambos grupos hicieron los mismos exámenes (Méndez et al. 2002).

El realizar la migración del curso de Agroindustria desde la modalidad tradicional a la modalidad virtual, hará que los estudiantes tengan una opción mucho más enriquecedora para llevar este curso, que les ofrezca posibilidades que de otra forma no podrían tener, además de 
motivarlos a aplicar la metacognición y el autoaprendizaje de una manera retadora y más satisfactoria (Pérez 2009).

Sin embargo, la Cátedra de Agroindustria no había empezado a estudiar este tema ni a aplicarlo en sus cursos hasta Octubre del 2008, cuando empezó a recibir capacitaciones en Web CT y la evaluación de los aprendizajes.

Debido a que es la primera vez que se da el curso bajo la modalidad virtual, es muy importante realizar un análisis profundo de los resultados, de la respuesta de los estudiantes y cómo está afectando el aprendizaje de los mismos.

La evaluación utilizada en el curso fue tanto tradicional como alternativa. Esto con el fin de retar el nivel de pensamiento del estudiante y retroalimentarlo de manera constante en su trabajo (López \& Hinojosa 2000).

Lo anterior es sumamente importante, ya que según Méndez \& Monge (2006), en teoría, en la UNED la función de los tutores es aclarar dudas, supervisar prácticas y guiar el aprendizaje, mientras que en la realidad, el alumnado costarricense nunca ha podido adaptarse a esta teoría debido a que llega a la UNED tras 12 años de aprendizaje presencial (Méndez \& Monge 2006).

Es por eso que la UNED está promoviendo fuertemente el desarrollo de la capacidad cognitiva del estudiante y la posibilidad de que tenga más opciones para demostrar su aprendizaje, a través del uso de la plataforma virtual (Pérez 2009).

Lo anterior se logra aplicando técnicas de evaluación del desempeño como mapas conceptuales, solución de casos, foros, proyectos de investigación y ensayos. Estas técnicas pueden ser validadas por instrumentos de evaluación como listas de cotejo, entrevistas, rúbricas o rangos (Pérez 2009). La universidad motiva a que se haga esto mezclado con técnicas tradicionales de evaluación como exámenes y quices.

La evaluación del desempeño es un método de evaluación del aprendizaje diseñada para recoger evidencias de los aprendizajes desarrollados por los educandos a lo largo del proceso educativo y con un valor numérico en la calificación final del curso (León 2007). Este método se utilizó en esta investigación.

Es muy importante reconocer el riesgo de inferir en la observación del desempeño y tomar las medidas necesarias para hacer más objetiva la evaluación. Para ello se deben seleccionar las técnicas más pertinentes (pruebas escritas, la observación o la resolución de problemas o una combinación de técnicas) (Pérez 2009).

A diferencia de la visión tradicional, aquí no se considera a la evaluación como dominio absoluto del ase- sor, sino que fomenta la participación de los educandos (autoevaluación y coevaluación) (León 2007).

Los objetivos fueron llevar al estudiante a interiorizar cada vez más la metacognición y enfocarse en documentar el crecimiento del individuo en cierto tiempo, en lugar de compararlo con los demás (Pérez 2009) e ir realizando la migración de los cursos tradicionales a la manera virtual (PAL 2008), PAL: Programa de Aprendizaje en línea de la Universidad Estatal a Distancia.

\section{METODOLOGÍA}

\section{Migración del curso de Agroindustria de modalidad tradicional a modalidad virtual}

Para realizar la migración del curso Agroindustria a la modalidad virtual, se siguieron una serie de pasos importantes:

a) Se reescribió la descripción curricular del curso, la cual era de 1980. Esto se realizó junto con los encargados de programa, la Comisión de la Escuela y la aprobación del Consejo de Escuela.

b) Se tomó el curso"Taller sobre el manejo de la plataforma Web CT" que da la universidad.

c) Se inició el diseño de curso en línea con base en la nueva descripción curricular, incluyendo artículos recientes tomados de internet y revistas científicas y se escribió un material complementario que explicaba los aspectos técnicos de cada tema. El diseño de este curso en Web CT se puede observar en el Apéndice 1.

d) Se afinaron todos los detalles para que el curso estuviera listo para el segundo cuatrimestre del año.

\section{Recolección de información sobre el impacto del curso en los estudiantes y su desempeño en el área de aprendizaje}

Durante el desarrollo del curso en línea, se llenó un registro en el que se fueron documentando todas las etapas del curso, la situación específica de cada estudiante, las calificaciones de los estudiantes obtenidas durante el curso y los resultados de la aplicación de los instrumentos para la medición del desempeño (Apéndices 2 y 3 ).

El estudiante tuvo que entregar una serie de tareas durante todo el cuatrimestre. Algunas de ellas tuvieron función formativa y otras, sumativa. Las tareas que se evaluaron de manera formativa, pertenecían todas al proyecto de investigación (Cuadro 2). Una vez que se le había dado al estudiante la retroalimentación necesaria para que 
corrigiera los borradores que enviaba (evaluación formativa), el estudiante procedía a entregar el avance correspondiente, el cual sí era evaluado de manera sumativa. Los estudiantes tuvieron que entregar cinco avances oficiales y una versión final, todas con un puntaje.

El resto de las tareas asignadas con puntaje fueron: dos foros, dos quices, una solución de caso y un examen comprensivo al final del cuatrimestre.

Las técnicas e instrumentos de evaluación del desempeño que entregamos a los tutores son, respectivamente, los de López \& Hinojosa (2000) y Pérez (2009).

Las técnicas de evaluación formativa y sumativa se tomaron de López \& Hinojosa (2000); y las directrices para determinar si existen diferencias significativas entre los resultados al inicio y al final del estudio de Loría \& Chaves (2002).

\section{Análisis de resultados y recomendaciones de mejora}

Durante el cuatrimestre se analizaron los resultados concretos que con el registro del Apéndice 1 se fueron recopilando. Todo esto hasta llegar al final del cuatrimestre, donde se vio el resultado final de cada estudiante y se concluyó sobre la evaluación de los aprendizajes.

Para concluir sobre la evaluación de los aprendizajes, se valoraron los métodos de enseñanza utilizados por medio de instrumentos de evaluación como listas de cotejo, entrevistas, rúbricas o rangos.

El primer instrumento de evaluación utilizado fue la entrevista, la cual se le realizó a los estudiantes para conocer su impresión general sobre el curso (Apéndice 6).

El segundo instrumento de evaluación es una lista de cotejo que sigue la técnica de observación la técnica descrita para las listas de corroboración. Este instrumento se utilizó para evaluar el desempeño del estudiante por parte del tutor durante el desarrollo del proyecto de investigación. Se pretendió dar a conocer cómo respondieron los estudiantes ante el reto de hacer un proyecto de investigación, la cual es la técnica de evaluación del desempeño más importante del curso (Apéndice 7).

El tercer instrumento utilizado fue una escala gráfica en donde se califica el concepto de cada estudiante de manera subjetiva (por parte del tutor), pero que dá una visión sobre cómo reaccionan los estudiantes ante la nueva modalidad (curso en línea) para tomar acción y corregir (Apéndice 9).

Las recomendaciones de mejora no solamente serán útiles al curso de Agroindustria que se dará en el II cuatrimestre del 2010, sino que pueden tomarse como ejemplo para el resto de los cursos de la Cátedra y la universidad.

\section{RESULTADOS}

\section{Rediseño del curso y migración a Web CT}

La descripción curricular del curso Agroindustria fue aprobada en el Consejo de marzo, 2009 de la Escuela de Ciencias Exactas y Naturales.

Una vez que se aprobó este curso, se procedió a montarlo en uno de los campos virtuales que tiene la UNED (Web CT) para el II cuatrimestre del 2009. En el Apéndice 1 se muestran las diferentes partes en las que se creó el curso en línea.

\section{Desempeño de los estudiantes, evaluación de los aprendizajes y medición del desempeño}

El desempeño de los estudiantes se midió en términos de cumplimiento, calificaciones y los resultados generados por los instrumentos de evaluación.

\section{Cumplimiento}

En el cuadro 1 se muestra el porcentaje de cumplimiento de los estudiantes en cada técnica que se les asignó con puntaje (evaluación sumativa). El porcentaje

\section{CUADRO 1}

Porcentaje de cumplimiento de entrega de técnicas de evaluación sumativa por parte de los estudiantes del curso de Agroindustria en línea impartido durante el PAC-2-2009

Técnica de evaluación

\begin{tabular}{cccccccccc}
\cline { 2 - 8 } & Foro 1 & Foro 2 & Quiz 1 & Quiz 2 & Caso & Avance 1 & Avance 2 & Avance 3 & Versión final \\
\cline { 2 - 10 } & 90 & 70 & 100 & 50 & 60 & 90 & 50 & 40 & 70 \\
\% = porcentaje de cumplimiento & & & & & & & & &
\end{tabular}


de cumplimiento de los estudiantes fue en promedio un $68,8 \%$.

Se puede observar como el porcentaje de cumplimiento para las técnicas con evaluación formativa es en general más bajo que el porcentaje de cumplimiento para las técnicas con evaluación sumativa. Sin embargo, de acuerdo a la prueba de contraste $Z$, con un $95 \%$ de confianza, se puede decir que no existen diferencias significativas entre ambos casos. Esto sugiere que los estudiantes están poniendo un empeño igual en las tareas que se les asignan para evaluarlos formativamente que en las tareas en las que se les dá una calificación sumativa y que las diferencias encontradas son producto del azar (Cuadro 2).

\section{Entrevista sobre la percepción de los estudiantes sobre el curso}

La evaluación realizada al curso por parte de los estudiantes a través de una entrevista muestra resultados muy importantes para los futuros cuatrimestres en los que se

\section{CUADRO 2}

Porcentaje de cumplimiento de entrega de técnicas de evaluación formativa por parte de los estudiantes del curso de Agroindustria en línea impartido durante el PAC-2-2009

\begin{tabular}{lc}
\hline \multicolumn{1}{c}{ Técnica de Evaluación } & $\% \mathrm{C}$ \\
\hline Marco teórico parte 1 & 50 \\
Problema & 50 \\
Objetivos & 50 \\
Justificación parte 1 & 50 \\
Quiz 1 & 100 \\
Recomendaciones de mejora parte 1 & 60 \\
Metodología & 70 \\
Marco Teórico parte 2 & 70 \\
Marco Teórico parte 3 & 70 \\
Resultados esperados & 40 \\
Marco Teórico 4 & 40 \\
Justificación parte 2 & 40 \\
Resultados obtenidos & 40 \\
Recomendaciones de mejora parte 2 & 40 \\
Marco teórico parte 5 & 50 \\
Justificación parte 3 & 40 \\
Quiz 2 & 50 \\
Versión final & 70 \\
\%C porcentaje de cumplimiento & \\
\hline
\end{tabular}

dé el curso y otros cursos en línea. A continuación se comentará cada sección y los resultados obtenidos.

Las respuestas de los estudiantes que contestaron la entrevista con respecto a su preferencia entre la modalidad virtual y la tradicional. No se pudo analizar el curso cuando se dio bajo la modalidad tradicional y después compararlo con esta nueva modalidad, debido a que la idea de la investigación nació justo antes de impartir el curso en línea. Se recomienda realizar otra investigación utilizando ambas modalidades para los otros cursos de la Cátedra (Cuadro 3).

CUADRO 3

Resultados obtenidos en la primera sección de la entrevista aplicada a los estudiantes en relación a la aceptación del curso de Agroindustria en línea durante el PAC-2-2009

\begin{tabular}{|c|c|c|}
\hline $\begin{array}{c}\text { Sección 1. Diferencia entre la modalidad } \\
\text { virtual y la tradicional }\end{array}$ & \% Sí & $\%$ No \\
\hline $\begin{array}{l}\text { ¿El curso Agroindustria fue más fácil para } \\
\text { usted que el resto de cursos que llevó bajo } \\
\text { la modalidad tradicional? }\end{array}$ & $33 \%$ & $66 \%$ \\
\hline $\begin{array}{l}\text { ¿Hubiera preferido tener tutorías } \\
\text { presenciales, al menos dos? }\end{array}$ & $33 \%$ & $66 \%$ \\
\hline $\begin{array}{l}\text { ¿Sintió que la atención por parte de su tutor } \\
\text { en línea fue mejor que cuando ha llevado } \\
\text { cursos de la manera tradicional? }\end{array}$ & $100 \%$ & $0 \%$ \\
\hline $\begin{array}{l}\text { ¿Pudo encontrar información más } \\
\text { actualizada en este curso que cuando ha } \\
\text { llevado cursos de la manera tradicional? }\end{array}$ & $100 \%$ & $0 \%$ \\
\hline
\end{tabular}

El cuadro 3 muestra que un 33\% los estudiantes encontraron más fácil el llevar el curso en línea a llevarlo de la manera tradicional, mientras que un $66 \%$ no lo encontró más fácil. Cabe recalcar que uno de los comentarios de las personas que respondió que sí, dijo que no era que había encontrado el curso más fácil, sino más cómodo.

Un $66 \%$ de las personas respondió que no necesitó tutorías presenciales, lo cual indica que el curso fue manejable al ser $100 \%$ en línea. Para las últimas dos preguntas de esta sección, el $100 \%$ de los entrevistados respondieron positivamente, lo cual indica que se sintieron más atendidos por parte del tutor al llevar el curso en línea y que el curso ofreció información más actualizada que la que hubieran encontrado si el curso se hubiera dado de manera tradicional.

El curso llenó las expectativas del 100\% de los estudiantes, que estuvo a la altura del nivel de licenciatura y que va 
a ser útil en la vida laboral de los estudiantes en un 100\%. Un 33\% de los estudiantes consideró que algunos temas se pudieron profundizar más, pero no mencionaron cuáles (Cuadro 4).

Los resultados de la entrevista en relación al Material Didáctico que la Cátedra preparó para impartir el curso en línea, tanto original de la Cátedra como artículos y lecturas actualizadas (Cuadro 5).

\section{CUADRO 4}

Resultados obtenidos en la segunda sección de la entrevista aplicada a los estudiantes en relación a la aceptación del curso de Agroindustria en línea durante el PAC-2-2009

\begin{tabular}{|c|c|c|}
\hline Sección 2. Temática y nivel & \% Sí & $\%$ No \\
\hline $\begin{array}{l}\text { ¿Considera que el curso llenó sus } \\
\text { expectativas en cuanto a contenido? }\end{array}$ & $100 \%$ & $0 \%$ \\
\hline $\begin{array}{l}\text { ¿Considera que hay temas que se pudieron } \\
\text { haber mencionado o profundizado más? } \\
\text { Justifique. }\end{array}$ & $33 \%$ & $66 \%$ \\
\hline $\begin{array}{l}\text { ¿En cuando al nivel de dificultad, cree usted } \\
\text { que hubo temas que le exigieron más de lo } \\
\text { que cree debería dar a nivel de Licenciatura? }\end{array}$ & $0 \%$ & $100 \%$ \\
\hline $\begin{array}{l}\text { ¿Cree que lo aprendido en el curso le va a } \\
\text { ser útil en su vida laboral? Explique }\end{array}$ & $100 \%$ & $0 \%$ \\
\hline
\end{tabular}

\section{CUADRO 5}

Resultados obtenidos en la tercera sección de la entrevista aplicada a los estudiantes en relación a la aceptación del curso de Agroindustria en línea durante el PAC-2-2009

\begin{tabular}{lcc}
\hline Sección 3. Material didáctico de la Cátedra & $\%$ Sí & $\%$ No \\
\hline $\begin{array}{l}\text { ¿El material didáctico diseñado por la } \\
\text { Cátedra llenó sus expectativas? }\end{array}$ & $100 \%$ & $0 \%$ \\
$\begin{array}{l}\text { ¿Los artículos científicos y las lecturas que } \\
\text { se le proporcionaron corresponden con lo } \\
\text { que usted esperaba aprender para poderlo }\end{array}$ & $100 \%$ & $0 \%$ \\
$\begin{array}{l}\text { utilizar en su campo laboral? } \\
\text { ¿El material que se le proporcionó fue }\end{array}$ & & \\
$\begin{array}{l}\text { suficientemente autoexplicativo para } \\
\text { poderlo entender sin la ayuda de tutorías? }\end{array}$ & $100 \%$ & $0 \%$ \\
\end{tabular}

El 100\% los estudiantes considera que el material didáctico estaba bien diseñado para llenar sus expectativas, que los artículos y lecturas fueron apropiados para el curso y que el material era autoexplicativo. Mucho de este material se incluyó en el libro de texto cuya versión preliminar se utilizará en el II cuatrimestre del 2010, por lo que es una buena base para pensar que es un material adecuado a las necesidades de los estudiantes (Cuadro 5).

Los resultados de la percepción del estudiante referente al proceso metacognitivo y autorregulativo al que tiene que enfrentarse para jugar un papel protagónico en el curso, el cual es uno de los objetivos más importantes para la universidad al dar un curso en línea (Cuadro 6).

El $100 \%$ de los estudiantes pudo automotivarse para poder llevar el curso al día, para cumplir con lo que el tutor le solicitó y que recibió la suficiente retroalimentación del mismo para sobrellevar sus dudas. Solamente un 33\% de los estudiantes consideró que las tareas asignadas por semana eran más de lo que hubieran podido manejar (Cuadro 6).

\section{CUADRO 6}

Resultados obtenidos en la cuarta sección de la entrevista aplicada a los estudiantes en relación a la aceptación del curso de Agroindustria en línea durante el PAC-2-2009

\begin{tabular}{lcc}
\hline \multicolumn{1}{c}{$\begin{array}{c}\text { Sección 4. Metacognición y } \\
\text { autorregulación }\end{array}$} & $\%$ Sí & $\%$ No \\
\hline $\begin{array}{l}\text { ¿El curso le permitió automotivarse } \\
\text { para poder seguir desde principio a fin, } \\
\text { cumpliendo todas las tareas que se le } \\
\text { asignaron? }\end{array}$ & $100 \%$ & $0 \%$ \\
$\begin{array}{l}\text { ¿Considera que el número de tareas } \\
\text { asignadas semana a semana fue más de lo } \\
\text { que hubiera podido manejar? }\end{array}$ & $33 \%$ & $66 \%$ \\
$\begin{array}{l}\text { ¿Pudo encontrar la manera de estudiar la } \\
\text { temática del curso para poder cumplir con } \\
\text { lo que el tutor esperaba de usted? }\end{array}$ & $100 \%$ & $0 \%$ \\
$\begin{array}{l}\text { Explique } \\
\text { ¿Cree que la retroalimentación recibida } \\
\text { por su tutor para con su proyecto de } \\
\text { investigación fue útil para su formación? }\end{array}$ & & \\
\end{tabular}

Los instrumentos de evaluación utilizados (foros, quices, examen, proyecto de investigación, estudio de caso y las visitas a la empresa), fueron herramientas útiles para el aprendizaje de los estudiantes. En el caso de los quices y el examen final, un $33 \%$ de los estudiantes comentó que no le fueron tan útiles para estudiar al día la materia (Cuadro 7).

\section{Proyecto de investigación}

Una de las técnicas de evaluación más importantes utilizadas para corroborar el nivel de aprendizaje de los estudiantes fue el Proyecto de Investigación. 
Es por lo anterior que se decidió aplicar una lista de corroboración en la que se evaluaran aspectos relacionados con la actitud del estudiante, con el compromiso con el proyecto y la comunidad o la empresa a la que ayudó con el mismo, a la veracidad de la información que presentó en el proyecto (aunque no se puede comprobar de manera directa, es fácil detectar cuando un estudiante no fue al campo a aplicar sus conocimientos). Esta lista es subjetiva, pero la consideramos de mucho valor para evaluar el efecto de esta técnica en el aprendizaje del estudiante.

Podemos observar que (ver Apéndice 9 para ver las preguntas que esta lista de corroboración tiene) las preguntas 3,6 y 7 es en donde hubo el menor cumplimiento de los estudiantes (Cuadro 8). De acuerdo a esta experiencia, se notó que los estudiantes no realizaban las correcciones que

\section{CUADRO 7}

Resultados obtenidos en la quinta sección de la entrevista aplicada a los estudiantes en relación a la aceptación del curso de Agroindustria en línea durante el PAC-2-2009

\begin{tabular}{|c|c|c|}
\hline Sección 5. Técnicas de evaluación del curso & \% Sí & $\%$ No \\
\hline $\begin{array}{l}\text { ¿Cree que los foros de discusión fueron } \\
\text { una herramienta útil para discutir } \\
\text { la implementación de proyectos } \\
\text { agroindustriales? }\end{array}$ & $100 \%$ & $0 \%$ \\
\hline $\begin{array}{l}\text { ¿Considera que los quices le ayudaron a } \\
\text { estudiar al día la materia? }\end{array}$ & $66 \%$ & $33 \%$ \\
\hline $\begin{array}{l}\text { ¿Cree que los avances del proyecto fueron } \\
\text { importantes para poderlo guiar en el camino } \\
\text { hasta la versión final? }\end{array}$ & $100 \%$ & $0 \%$ \\
\hline $\begin{array}{l}\text { ¿Cree que el examen final fue importante } \\
\text { para afianzar sus conocimientos? Explique }\end{array}$ & $66 \%$ & $33 \%$ \\
\hline $\begin{array}{l}\text { ¿Considera la solución de caso una técnica } \\
\text { útil para su formación profesional? Explique }\end{array}$ & $100 \%$ & $0 \%$ \\
\hline $\begin{array}{l}\text { ¿Considera que el visitar una empresa } \\
\text { diferentes veces y la implementación de un } \\
\text { proyecto le ayudaron a aclarar su visión de } \\
\text { su futuro campo laboral? }\end{array}$ & $100 \%$ & $0 \%$ \\
\hline
\end{tabular}

el tutor les hacía cuando entregaban borradores de cada parte del proyecto, por falta de tiempo o porque quizás no creyeran que les iba a generar problemas; pero esto definitivamente afectó en la evaluación sumativa del proyecto.

Se puede observar (Cuadro 9) que menos de la mitad de los estudiantes mostraron un rendimiento que se pueda considerar alto (lo que fue catalogado como "Siempre") y que aproximadamente la otra mitad se catalogó entre "Frecuentemente" $y$ "Ocasionalmente".

\section{DISCUSIÓN}

El iniciar un curso en línea por primera vez fue una experiencia que todas las Cátedras de la Universidad Estatal a Distancia están teniendo tarde o temprano y sumamente importante para el proceso de aprendizaje tanto de los estudiantes como de los tutores y encargados de cátedra. Sin embargo, el cambio en la evaluación de los aprendizajes, al ser un cambio de actitud, de "pensar fuera de la caja", es un cambio gradual que no se va a conseguir con la primera vez que se imparte el curso. Este estudio es apenas una pincelada en la cual se rescatan aquellos detalles más importantes a cambiar para la segunda vez que se dé el curso, en el II cuatrimestre del 2010.

Si la metacognición, que es uno de los objetivos más importantes de un curso en línea, permite a los estudiantes tomar conciencia de su propio aprendizaje y visualizar en qué lo puede aplicar (Bordas \& Cabrera 2001), de acuerdo a los resultados obtenidos se podría decir que en general hubo una buena aplicación de esta habilidad por parte de los estudiantes, ya que lograron interiorizar los conocimientos que se esperaba adquirieran de manera independiente.

Considerando la experiencia del curso en línea y la aplicación de los instrumentos para la medición del desempeño, se observó que la razón por la cual los estudiantes se interesaron en entregar las tareas evaluadas de manera formativa referentes al proyecto de investigación fue la retroalimentación que se les iba a dar, la cual iba a lograr que obtuvieran una mejor calificación en cada avance del

\section{CUADRO 8}

Porcentaje de estudiantes que obtuvieron un resultado positivo al aplicar la lista de corroboración para evaluar su desempeño durante la elaboración del Proyecto de Investigación del curso de Agroindustria en línea durante el PAC-2-2009

\begin{tabular}{|c|c|c|c|c|c|c|c|c|}
\hline & \multicolumn{8}{|c|}{$N^{\circ}$ pregunta } \\
\hline & 1 & 2 & 3 & 4 & 5 & 6 & 7 & 8 \\
\hline Resultado positivo & $70 \%$ & $90 \%$ & $60 \%$ & $70 \%$ & $70 \%$ & $60 \%$ & $60 \%$ & $100 \%$ \\
\hline
\end{tabular}




\section{CUADRO 9}

Resultados obtenidos al aplicar la escala gráfica a los estudiantes del curso de Agroindustria en línea durante el PAC-2-2009 para medir el desempeño de los mismos en el curso

\begin{tabular}{|c|c|c|c|c|c|}
\hline \multirow[b]{2}{*}{ Pregunta } & \multicolumn{5}{|c|}{ Desempeño de los estudiantes } \\
\hline & Nunca & Rara vez & Ocasionalmente & Frecuentemente & Siempre \\
\hline $\begin{array}{l}\text { ¿El alumno tuvo una participación } \\
\text { suficiente todas las semanas en todas las } \\
\text { actividades realizadas? }\end{array}$ & 1 & 1 & 2 & 3 & 3 \\
\hline $\begin{array}{l}\text { ¿El estudiante aporta ideas valiosas al tema } \\
\text { en cada evaluación que se realizó? }\end{array}$ & 1 & 0 & 3 & 4 & 2 \\
\hline $\begin{array}{l}\text { ¿ El estudiante puede interiorizar aplicando } \\
\text { la metacognición, los conocimientos del } \\
\text { curso? }\end{array}$ & 1 & 0 & 0 & 4 & 5 \\
\hline $\begin{array}{l}\text { ¿El estudiante aporta de manera } \\
\text { significativa en la zona rural en donde está } \\
\text { realizando su trabajo de investigación? }\end{array}$ & 1 & 0 & 0 & 0 & 9 \\
\hline
\end{tabular}

proyecto. Esto se evidencia en los resultados de la entrevista virtual que se realizó a los estudiantes con respecto a su impresión con respecto al curso.

A pesar de que no se encontraron diferencias significativas en el empeño puesto por los estudiantes en la evaluación sumativa y la formativa, sí se pudo observar que a excepción de los más aplicados, los estudiantes siguen limitando sus esfuerzos a aquello que va a tener significancia en su calificación final, en vez de ver el proceso como un todo que les permita tomar consciencia de su propio aprendizaje y corroborar su aprendizaje, como lo dice el modelo pedagógico de la UNED.

Lo anterior también puede mostrar que la corroboración del progreso del aprendizaje de los estudiantes por parte del tutor no se pudo realizar tan profundamente como la orientación de curso lo ambicionaba, ya que después de realizar retroalimentación en los trabajos realizados por los estudiantes, muchos no enviaban el trabajo corregido de la manera en la que se solicitaba, sino que de una manera parcial, lo cual podía implicar que no había tanto interés por los estudiantes de mejorar su trabajo, que no entendían las observaciones del tutor y no preguntaban al respecto o que no las consideraron importantes.
Sin embargo, de acuerdo a la entrevista realizada a los estudiantes, se puede observar que todos los estudiantes consideraron que la retroalimentación recibida por su tutor fue útil para su formación, lo cual puede que implique que los estudiantes no tengan tanto tiempo para hacer este tipo de correcciones por razones de estudio o trabajo y no necesariamente que no les interese realizarlas.

Además de ayudar al estudiante a aprender la materia que está viendo en el curso, la evaluación del proceso o formativa debe también ser funcional, ya que el estudiante la puede encontrar útil para su campo laboral al poder visualizar situaciones reales (Bautista et al. 2009). La entrevista realizada a los estudiantes, tanto el punto $b$ de la sección 3 como los puntos e y $\mathrm{f}$ de la sección 5 fueron respondidos de manera afirmativa por el $100 \%$ de los estudiantes y en todos se preguntaba si el curso fue enfocado de manera tal que se pudieran aplicar y visualizar conceptos relacionados con el campo laboral, lo cual implica que uno de los objetivos más importantes del curso se cumplió.

La lista de revisión utilizada para evaluar el desempeño de los estudiantes (Cuadro 8), muestra este mismo aspecto y lo importante a resaltar es que las personas que no demostraron darle seguimiento a la retroalimentación del 
profesor, son las que tuvieron notas más bajas. Según Bou et al. (2003), las tareas evaluativas deben provocar curiosidad, ser transferibles, progresivas en dificultad y acumulativas respecto a lo que se evalúa y potenciar el diálogo entre estudiantes y profesores. Alguno de estos factores pudo haber faltado en la retroalimentación dada por el tutor. Se podría intentar mejorar este aspecto la próxima vez que se de el curso y así lograr una mejor respuesta de parte de los estudiantes.

La evaluación en grupo es una técnica que no se utilizó esta vez en el curso y se podría experimentar la próxima vez que se imparta ya que realiza un aporte pedagógico transversal y al mismo tiempo hace que el estudiante pueda aplicar sus conocimientos para simular situaciones laborales de una manera más clara (Bautista et al. 2009).

\section{AGRADECIMIENTOS}

Agradezco a los estudiantes de la primera promoción del curso Agroindustria, que lo cursaron durante el segundo cuatrimestre del 2009, por su colaboración, interés y entusiasmo que logró enriquecer el curso para hacerlo mucho mejor para el siguiente cuatrimestre. De igual forma, agradezco el apoyo que la ECEN dio en este proyecto, en especial a Olman Díaz, Luis Montero Castro y Harold Arias y a la Vicerrectoría de Investigación, en especial a Kathya Calderón y Julián Monge-Nájera.

\section{REFERENCIAS}

Barrón, H. 2004. La educación superior en línea en México. (http://www.cronica.com.mx/nota.php?idc=145189; consultado 2 de Julio, 2009).

Bautista, G., F. Borges \& A. Forés. 2009. Didáctica universitaria en entornos virtuales. Anaya, Madrid, España.

Bordas, M. \& F. Cabrera. 2001. Estrategias de evaluación de los aprendizajes centradas en el proceso. (http://docs.google.com/viewer?a=v\&q=cache:tKOll9KGxqcJ:psicompet entes.files.wordpress.com/2008/06/recension-de-teresa. pdf+Bordas+y+Cabrera\&hl=es\&gl=cr\&sig=AHIEtbSCTFhx D5mf4sFygYtObdvluae-ew; consultado 4 Febrero, 2010).
Bou, G., T. Cascudo \& L. Huguet. 2003. E-learning. Anaya, Madrid, España.

Fonseca, P. 2009. Científicos costarricenses desaprovechan internet.(http://www.nacion.com/ln_ee/2009/abril/28/aldea1947135.html; consultado 2 de Julio, 2009)

León, C. 2007. Evaluación alternativa. Instituto Nacional para la Educación de Adultos. (http://uned.blackboard.com/webct/urw/lc5116001.tp0/cobaltMainFrame.dowebct; consultado 15 de Julio, 2009).

López, S. \& E. Hinojosa. 2000. Evaluación del aprendizaje: alternativas y nuevos desarrollos. Trillas, México D.F., México.

Loría, M. \& O. Chaves. 2002. Diseño de ensayos y experimentos. EUNED, San José, Costa Rica.

Méndez, V., J. Monge \& R. Rivas. 2002. La evolución de los laboratorios virtuales durante una experiencia de cuatro años con estudiantes a distancia. XI Congreso Internacional UNED 2002: sobre Tecnología y Educación a Distancia, 5-8 de Noviembre, San José, Costa Rica.

Méndez, V. \& J. Monge. 2006. Las TIC en un entorno latinoamericano de educación a distancia: la experiencia de la UNED de Costa Rica. Publicación en línea. Año V, № 15. Murcia, España.

Méndez, V. \& J. Monge. 2007. Ventajas y desventajas de usar laboratorios virtuales en educación a distancia: la opinión del estudiantado en un proyecto de seis años de duración. Revista de Educación 31: 91-108

Miranda, J. 2007. Educación en línea: una opción universitaria. (http://www.uvs.sld.cu/Members/leslierdguez/educacion-en-linea-una-opcion-universitaria/; consultado el 2 de Julio, 2009)

PAL (Programa de Aprendizaje en línea). 2008. Taller del manejo de la plataforma Web CT. Programa de aprendizaje en línea. San José, Costa Rica.

Pérez, J. 2009. Curso evaluación de los aprendizajes en entornos virtuales. Comunicación personal. UNED. San José, Costa Rica.

Salas, M. 2008. Organización y diseño de curso en línea, múltiples dimensiones: una propuesta del Centro de Capacitación en Educación a Distancia de la UNED -Costa Rica. (http://www.virtualeduca.info/forumveduca/index.php? Itemid=26\&id=198\&option=com _ content\&task=view; consultado 2 de Julio, 2009). 


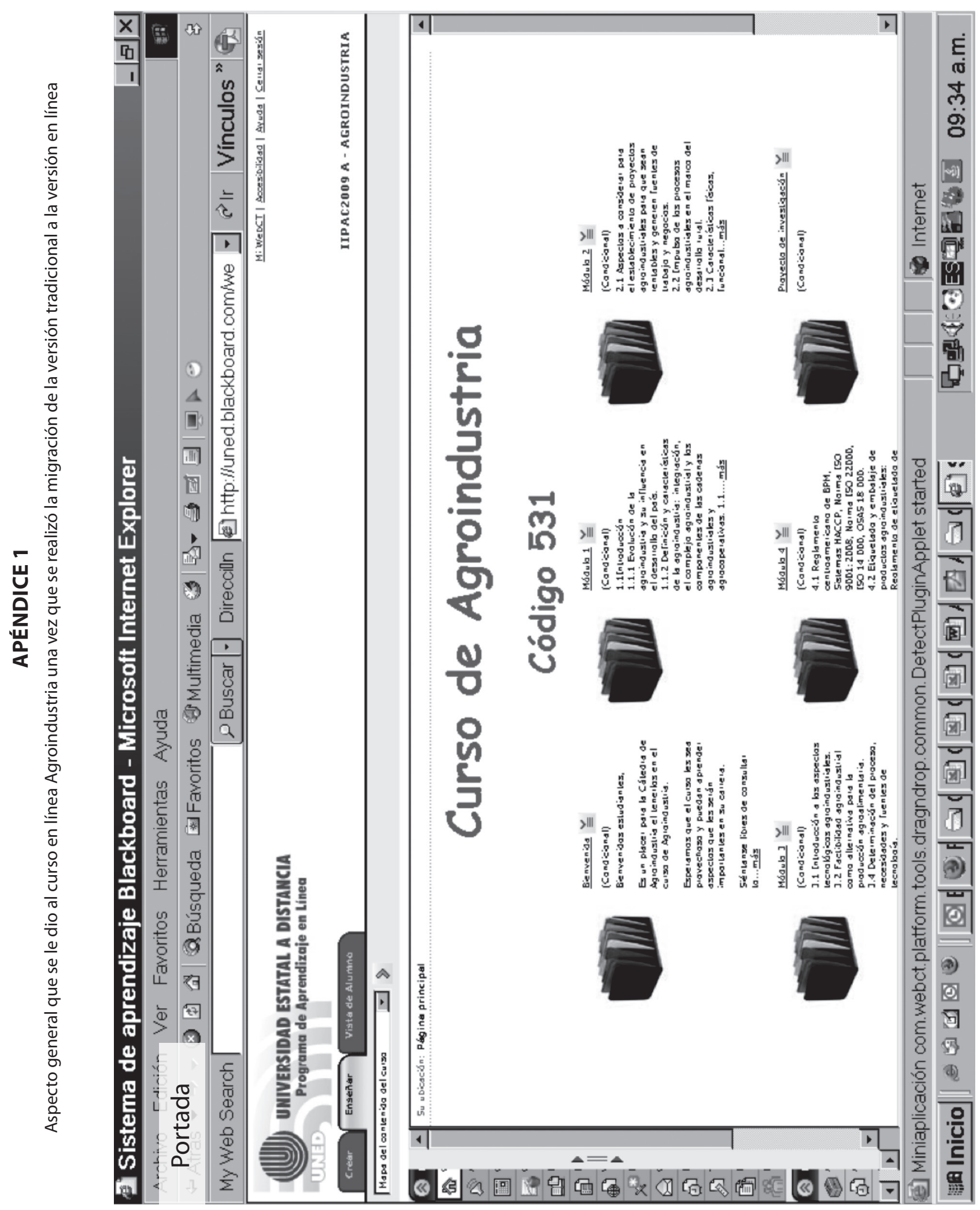




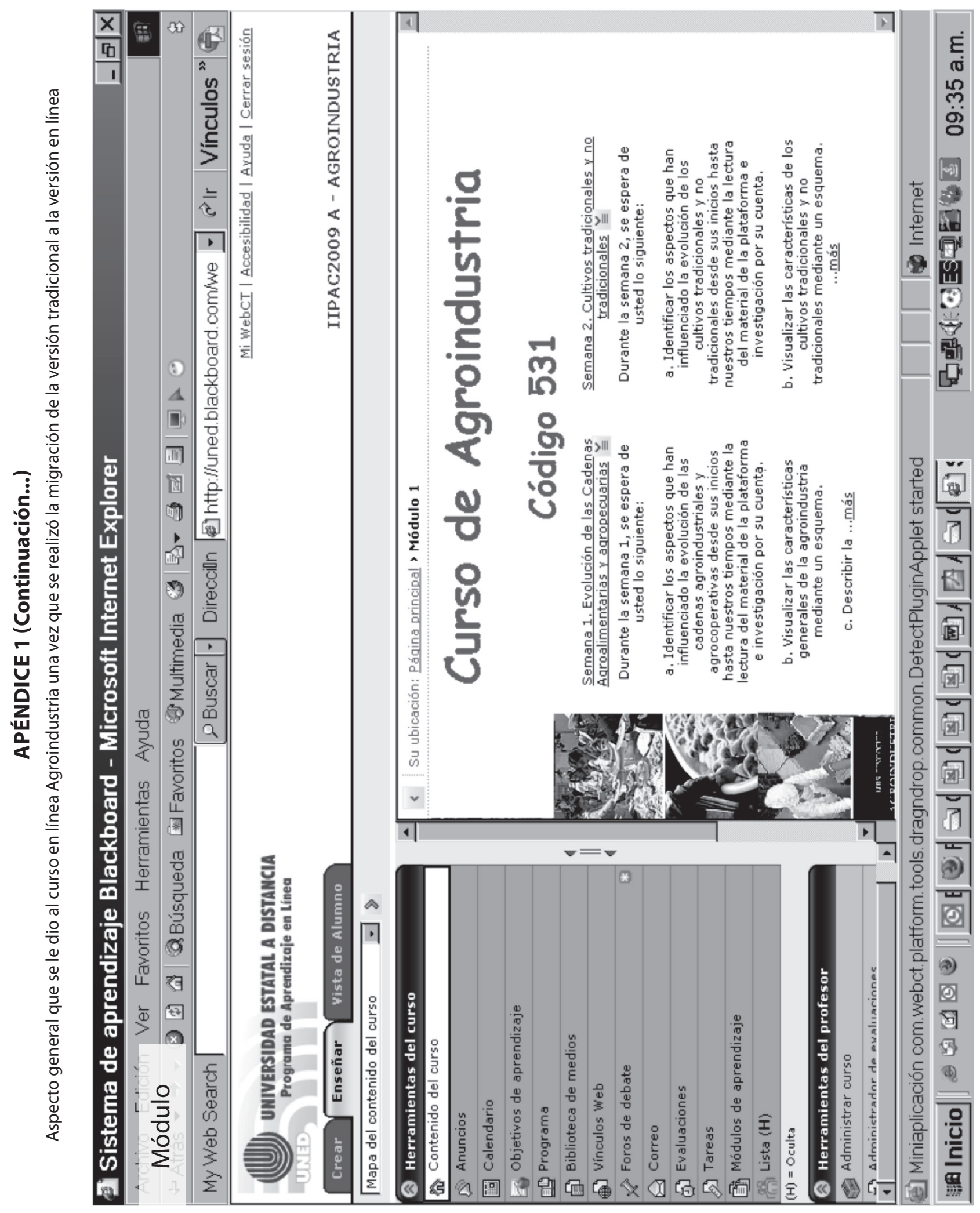




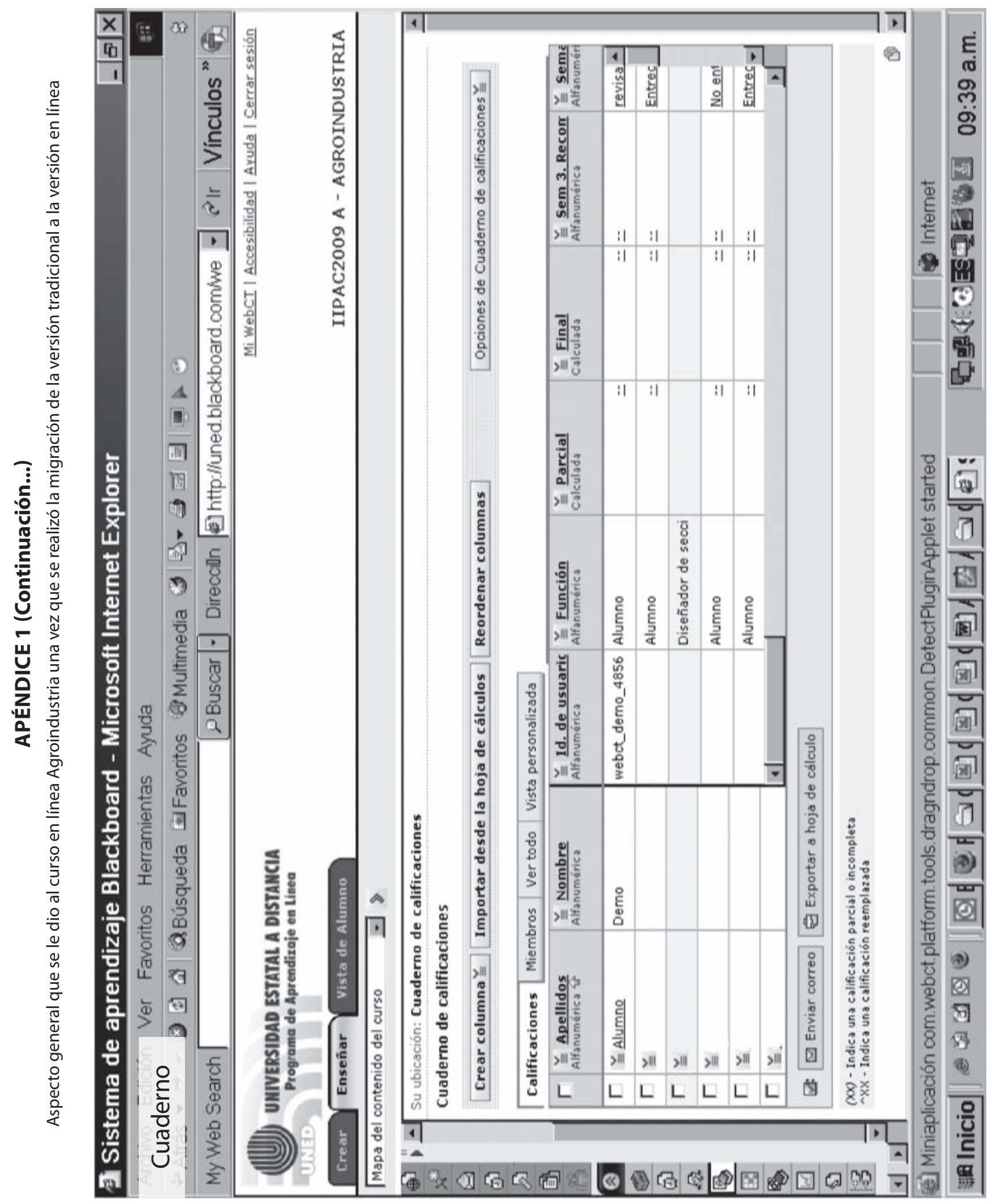



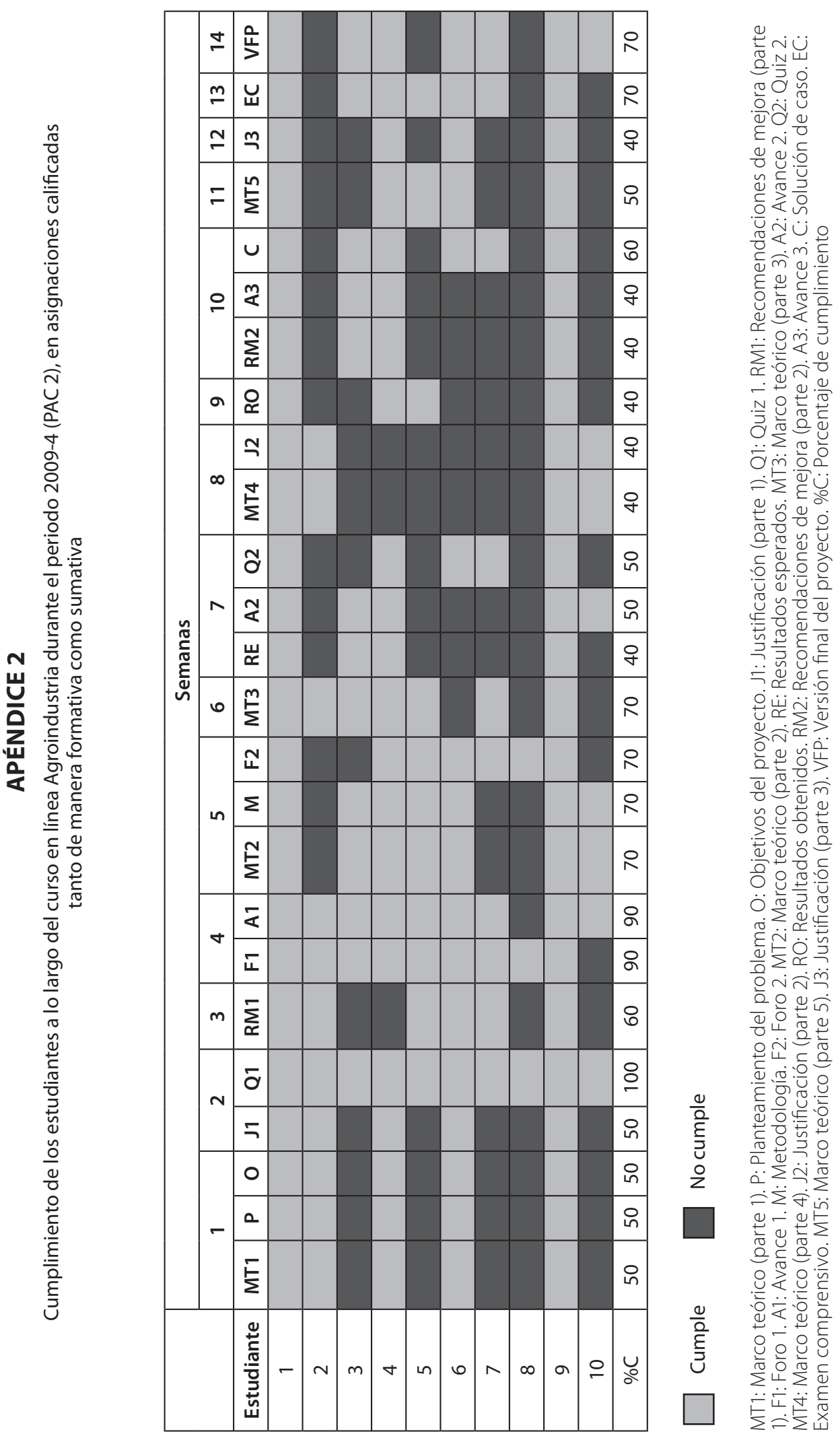


\section{APÉNDICE 3}

Registro de la situación de los estudiantes a lo largo del curso en línea Agroindustria durante el Il cuatrimestre del 2009

\begin{tabular}{|c|c|}
\hline Estudiante & Observaciones \\
\hline 1 & Inició bien. No entregó segundo avance porque tuvo accidente laboral, pero se reintegró. \\
\hline 2 & Ha estado constante durante el curso, excepto en el segundo avance. \\
\hline 3 & Estuvo bien al principio. No ha justificado sus altibajos. \\
\hline 4 & Su desempeño es excelente. \\
\hline 5 & Su desempeño es excelente. \\
\hline 6 & Tuvo un robo en su casa por lo que se le dio más tiempo para presentar los trabajos, pero aún así sigue con altibajos. \\
\hline 7 & $\begin{array}{l}\text { Ha entregado los trabajos a tiempo, excepto el primer avance. Se le dio tiempo un semana después de la fecha para } \\
\text { que lo entregara con base } 5 \% .\end{array}$ \\
\hline 8 & $\begin{array}{l}\text { Estaba en Nicaragua, por eso no se pudo contactar, pero la idea es que reinicie el curso y se le está dando la } \\
\text { oportunidad de entregar los trabajos y el primer avance con base } 5 \% \text {. Los foros los entregó en un ensayo, base } 10 \\
\text { cada uno. }\end{array}$ \\
\hline 9 & $\begin{array}{l}\text { No entregó a tiempo, si participó en Foro 1, pero no envió el primer avance a tiempo. Se le dio tiempo hasta el } \\
\text { domingo con base } 5 \% \text {. }\end{array}$ \\
\hline 10 & Se retiró del curso. Es privado de libertad. \\
\hline 11 & Su teléfono no responde. Se le ha tratado de llamar durante el curso. Inició bien pero dejó de mandar los trabajos. \\
\hline 12 & Retiró porque tenía que darle prioridad a otras materias. \\
\hline 13 & Retiró. No fue posible localizarlo. \\
\hline
\end{tabular}




\section{APÉNDICE 4}

Rúbrica (escala de calificación) utilizada para evaluar el desempeño de los estudiantes durante el curso Agroindustria en línea del PAC 2009 en la solución de caso: "Servicio al cliente"

\begin{tabular}{|c|c|c|c|c|}
\hline \multirow{2}{*}{ Indicadores } & \multicolumn{4}{|l|}{ Escala de valores } \\
\hline & 1 & 2 & 3 & 4 \\
\hline $\begin{array}{l}\text { Identifica, selecciona y plantea } \\
\text { correctamente el problema }\end{array}$ & $\begin{array}{l}\text { Identifica algunos } \\
\text { problemas }\end{array}$ & $\begin{array}{c}\text { Selecciona algunos } \\
\text { problemas }\end{array}$ & $\begin{array}{c}\text { Identifica, selecciona y } \\
\text { plantea los problemas } \\
\text { pero no de manera clara } \\
\text { o completa }\end{array}$ & $\begin{array}{c}\text { Identifica, selecciona y } \\
\text { plantea los problemas } \\
\text { de manera clara y } \\
\text { completa }\end{array}$ \\
\hline $\begin{array}{c}\text { Busca y plantea alternativas de } \\
\text { solución }\end{array}$ & $\begin{array}{c}\text { Busca alternativas } \\
\text { de solución (sólo las } \\
\text { menciona) }\end{array}$ & $\begin{array}{l}\text { Menciona y plantea } \\
\text { alternativas de } \\
\text { solución pero no } \\
\text { todas las necesarias } \\
\text { o algunas } \\
\text { incorrectas. }\end{array}$ & \begin{tabular}{|c|} 
Menciona y plantea \\
alternativas de solución \\
pero no todas las \\
necesarias.
\end{tabular} & $\begin{array}{c}\text { Menciona y plantea } \\
\text { todas las alternativas } \\
\text { de solución necesarias } \\
\text { y correctas para } \\
\text { solucionar el } \\
\text { problema. }\end{array}$ \\
\hline $\begin{array}{c}\text { Compara y analiza las alternativas } \\
\text { contemplando ventajas, } \\
\text { desventajas, consecuencias y } \\
\text { valores involucrados. }\end{array}$ & $\begin{array}{c}\text { Compara las } \\
\text { alternativas pero no } \\
\text { analiza }\end{array}$ & $\begin{array}{l}\text { Compara las } \\
\text { alternativas } \\
\text { analizando sólo } \\
\text { ventajas y } \\
\text { desventajas }\end{array}$ & \begin{tabular}{|c|} 
Compara las \\
alternativas analizando \\
ventajas, desventajas, \\
consecuencias y \\
valores, pero no acierta \\
en su respuesta \\
\end{tabular} & $\begin{array}{c}\text { Compara las } \\
\text { alternativas analizando } \\
\text { ventajas, desventajas, } \\
\text { consecuencias y } \\
\text { valores, y su respuesta } \\
\text { es válida. } \\
\end{array}$ \\
\hline $\begin{array}{l}\text { Plantea suposiciones que son } \\
\text { lógicas, basadas en su } \\
\text { conocimiento. }\end{array}$ & $\begin{array}{l}\text { No plantea } \\
\text { suposiciones }\end{array}$ & $\begin{array}{l}\text { Las suposiciones } \\
\text { que plantea no son } \\
\text { válidas }\end{array}$ & $\begin{array}{l}\text { Plantea suposiciones } \\
\text { pero no las suficientes }\end{array}$ & $\begin{array}{c}\text { Plantea todas las } \\
\text { suposiciones } \\
\text { necesarias para llegar } \\
\text { a la solución del } \\
\text { problema. } \\
\end{array}$ \\
\hline $\begin{array}{l}\text { Toma una decisión y fundamenta } \\
\text { ésta }\end{array}$ & $\begin{array}{l}\text { No toma una } \\
\text { decisión }\end{array}$ & $\begin{array}{c}\text { No toma una } \\
\text { decisión aunque dá } \\
\text { algunos } \\
\text { fundamentos }\end{array}$ & $\begin{array}{l}\text { Toma una decisión pero } \\
\text { su fundamento no es } \\
\text { suficientemente fuerte }\end{array}$ & $\begin{array}{l}\text { Toma una decisión y } \\
\text { su fundamento es el } \\
\text { correcto. }\end{array}$ \\
\hline $\begin{array}{l}\text { Genera una propuesta de acción } \\
\text { con pasos a seguir. }\end{array}$ & $\begin{array}{c}\text { No genera una } \\
\text { propuesta de acción }\end{array}$ & $\begin{array}{l}\text { Su propuesta de } \\
\text { acción no tiene } \\
\text { pasos a seguir }\end{array}$ & $\begin{array}{c}\text { Su propuesta de acción } \\
\text { tiene pasos a seguir } \\
\text { pero no son los } \\
\text { correctos } \\
\end{array}$ & $\begin{array}{l}\text { Su propuesta de } \\
\text { acción tiene pasos a } \\
\text { seguir y son los } \\
\text { correctos. }\end{array}$ \\
\hline \multicolumn{5}{|l|}{ Puntaje obtenido: } \\
\hline Porcentaje: & & & & \\
\hline
\end{tabular}

Basado en: Ramírez, J \& Santander, E. 2003. Instrumentos de evaluación a través de competencias. Santiago, Chile. Consulta realizada en internet el 6 de Noviembre, 2008. Disponible en: http://competencias.iespana.es/instrumentos\%20de\%20evaluacion\%20a\%20traves\%20de\%20competencias.pdf. 


\section{APÉNDICE 5}

Rúbrica (escala de calificación) utilizada para evaluar el desempeño de los estudiantes durante el curso Agroindustria en línea del PAC 2009 en los Foros de discusión

\begin{tabular}{|c|c|c|c|c|}
\hline Criterios & \multicolumn{4}{|c|}{ Escala de calificación } \\
\hline $\begin{array}{l}\text { Número de } \\
\text { participaciones }\end{array}$ & $\begin{array}{l}\text { Participa de la manera } \\
\text { correcta, con citas } \\
\text { bibliográficas y analizando } \\
\text { según lo solicitado en al } \\
\text { menos } 5 \text { oportunidades } \\
\text { espaciadas (principio, } \\
\text { mediados y final) durante la } \\
\text { semana. }\end{array}$ & $\begin{array}{l}\text { Participa de la manera } \\
\text { correcta, con citas } \\
\text { bibliográficas y analizando } \\
\text { según lo solicitado en } 5 \\
\text { oportunidades diferentes } \\
\text { pero no espaciadas (sólo } \\
\text { al inicio y al final, sólo a } \\
\text { mediados, sólo a mediados y } \\
\text { al final, etc). } \\
5\end{array}$ & $\begin{array}{l}\text { Participa analizando según } \\
\text { lo solicitado en al menos } 5 \\
\text { oportunidades espaciadas } \\
\text { (principio, mediados y final) } \\
\text { durante la semana, pero no } \\
\text { adjunta citas bibliográficas. }\end{array}$ & $\begin{array}{l}\text { Participa de la manera } \\
\text { correcta, con citas } \\
\text { bibliográficas y analizando } \\
\text { según lo solicitado en } \\
\text { menos de } 5 \text { oportunidades } \\
\text { diferentes pero no } \\
\text { espaciadas (sólo al inicio } \\
\text { y al final, sólo a mediados, } \\
\text { sólo a mediados y al final, } \\
\text { etc). } \\
0\end{array}$ \\
\hline $\begin{array}{l}\text { Conocimiento del } \\
\text { tema }\end{array}$ & $\begin{array}{l}\text { Todas sus participaciones } \\
\text { reflejan que estudió a } \\
\text { fondo la aplicación de las } \\
\text { operaciones unitarias, los } \\
\text { métodos de conservación } \\
\text { y la determinación de la } \\
\text { vida útil en la incorporación } \\
\text { de valor agregado a la } \\
\text { producción primaria, } \\
\text { soportándolo mediante } \\
\text { citas bibliográficas, } \\
\text { ejemplos y comentarios con } \\
\text { sentido. } \\
25\end{array}$ & $\begin{array}{l}\text { La mayoría de sus } \\
\text { participaciones reflejan que } \\
\text { estudió a fondo la aplicación } \\
\text { de las operaciones unitarias, } \\
\text { los métodos de conservación } \\
\text { y la determinación de la } \\
\text { vida útil en la incorporación } \\
\text { de valor agregado a la } \\
\text { producción primaria, } \\
\text { soportándolo mediante citas } \\
\text { bibliográficas, ejemplos y } \\
\text { comentarios con sentido. } \\
15\end{array}$ & $\begin{array}{l}\text { Sólo en algunas de sus } \\
\text { participaciones reflejan que } \\
\text { estudió a fondo la aplicación } \\
\text { de las operaciones } \\
\text { unitarias, los métodos } \\
\text { de conservación y la } \\
\text { determinación de la vida útil } \\
\text { en la incorporación de valor } \\
\text { agregado a la producción } \\
\text { primaria, soportándolo } \\
\text { mediante citas bibliográficas, } \\
\text { ejemplos y comentarios con } \\
\text { sentido. } \\
10\end{array}$ & $\begin{array}{l}\text { Sólo una o ninguna } \\
\text { de sus participaciones } \\
\text { reflejan que estudió a } \\
\text { fondo la aplicación de las } \\
\text { operaciones unitarias, los } \\
\text { métodos de conservación } \\
\text { y la determinación de la } \\
\text { vida útil en la incorporación } \\
\text { de valor agregado a la } \\
\text { producción primaria, } \\
\text { soportándolo mediante } \\
\text { citas bibliográficas, } \\
\text { ejemplos y comentarios } \\
\text { con sentido. } \\
5\end{array}$ \\
\hline $\begin{array}{l}\text { Determina la } \\
\text { metodología a seguir } \\
\text { para experimentar } \\
\text { las operaciones } \\
\text { unitarias, los métodos } \\
\text { de conservación y } \\
\text { la determinación } \\
\text { de la vida útil en } \\
\text { una agroindustria, } \\
\text { recopilar información } \\
\text { e interpretarla }\end{array}$ & $\begin{array}{l}\text { Todas sus participaciones } \\
\text { reflejan que determina } \\
\text { correctamente los } \\
\text { aspectos a considerar } \\
\text { para la aplicación de estas } \\
\text { tecnologías mediante citas } \\
\text { bibliográficas y ejemplos. }\end{array}$ & $\begin{array}{l}\text { Todas sus participaciones } \\
\text { reflejan que determina } \\
\text { correctamente los aspectos a } \\
\text { considerar para la aplicación } \\
\text { de estas tecnologías } \\
\text { mediante citas bibliográficas } \\
\text { y ejemplos. }\end{array}$ & $\begin{array}{l}\text { Todas sus participaciones } \\
\text { reflejan que determina } \\
\text { correctamente los aspectos a } \\
\text { considerar para la aplicación } \\
\text { de estas tecnologías } \\
\text { mediante citas bibliográficas } \\
\text { y ejemplos. }\end{array}$ & $\begin{array}{l}\text { Todas sus participaciones } \\
\text { reflejan que determina } \\
\text { correctamente los } \\
\text { aspectos a considerar } \\
\text { para la aplicación de estas } \\
\text { tecnologías mediante citas } \\
\text { bibliográficas y ejemplos. }\end{array}$ \\
\hline $\begin{array}{l}\text { Discute la tecnología } \\
\text { específica que quiere } \\
\text { experimentar en la } \\
\text { fábrica o empresa en } \\
\text { la que quiere realizar } \\
\text { su proyecto y analiza } \\
\text { pros y contras y } \\
\text { maneras de aplicarla. }\end{array}$ & $\begin{array}{l}\text { En todas sus participaciones } \\
\text { logró plasmar de una } \\
\text { manera acertada cómo va a } \\
\text { experimentar la tecnología } \\
\text { en su proyecto para mejorar } \\
\text { un proceso o problema } \\
\text { específico. } \\
15\end{array}$ & $\begin{array}{l}\text { En todas sus participaciones } \\
\text { logró plasmar de una } \\
\text { manera acertada cómo va a } \\
\text { experimentar la tecnología } \\
\text { en su proyecto para mejorar } \\
\text { un proceso o problema } \\
\text { específico. } \\
10\end{array}$ & $\begin{array}{l}\text { En todas sus participaciones } \\
\text { logró plasmar de una } \\
\text { manera acertada cómo va a } \\
\text { experimentar la tecnología } \\
\text { en su proyecto para mejorar } \\
\text { un proceso o problema } \\
\text { específico. } \\
5\end{array}$ & $\begin{array}{l}\text { En todas sus } \\
\text { participaciones logró } \\
\text { plasmar de una manera } \\
\text { acertada cómo va a } \\
\text { experimentar la tecnología } \\
\text { en su proyecto para } \\
\text { mejorar un proceso o } \\
\text { problema específico. } \\
2,5\end{array}$ \\
\hline $\begin{array}{l}\text { Uso correcto de la } \\
\text { netiqueta }\end{array}$ & $\begin{array}{l}\text { En todas sus intervenciones } \\
\text { utiliza correctamente el } \\
\text { idioma, precisión, concisión, } \\
\text { claridad, brevedad y se } \\
\text { muestra respetuoso con los } \\
\text { demás compañeros. } \\
10\end{array}$ & $\begin{array}{l}\text { En la mayoría de sus } \\
\text { intervenciones utiliza } \\
\text { correctamente el idioma, } \\
\text { precisión, concisión, claridad, } \\
\text { brevedad y se muestra } \\
\text { respetuoso con los demás } \\
\text { compañeros. } \\
5\end{array}$ & $\begin{array}{l}\text { Sólo en algunas de sus } \\
\text { intervenciones utiliza } \\
\text { correctamente el idioma, } \\
\text { precisión, concisión, } \\
\text { claridad, brevedad y se } \\
\text { muestra respetuoso con los } \\
\text { demás compañeros. } \\
2,5\end{array}$ & $\begin{array}{l}\text { Sólo en una o ninguna de } \\
\text { sus intervenciones utiliza } \\
\text { correctamente el idioma, } \\
\text { precisión, concisión, } \\
\text { claridad, brevedad y se } \\
\text { muestra respetuoso con los } \\
\text { demás compañeros. } \\
0\end{array}$ \\
\hline \multicolumn{5}{|l|}{ PUNTAJE OBTENIDO } \\
\hline PUNTAJE TOTAL & & & & \\
\hline
\end{tabular}




\section{APÉNDICE 6}

Entrevista en línea utilizada para evaluar la percepción de los estudiantes sobre el curso

\section{Entrevista para conocer la percepción del estudiante sobre el curso Agroindustria, impartido en línea durante el II 2009}

Persona que llena la entrevista:

Fecha:

\section{Instrucciones}

A continuación se le presentan una serie de preguntas separadas en seis temas diferentes. Para cada tema se colocaron preguntas de marque con $\mathrm{x}$. Usted debe marcar la respuesta correcta según su percepción del curso de Agroindustria. Es muy importante que justifique cada una de sus respuestas. Sea especifico.

\section{Entrevista}

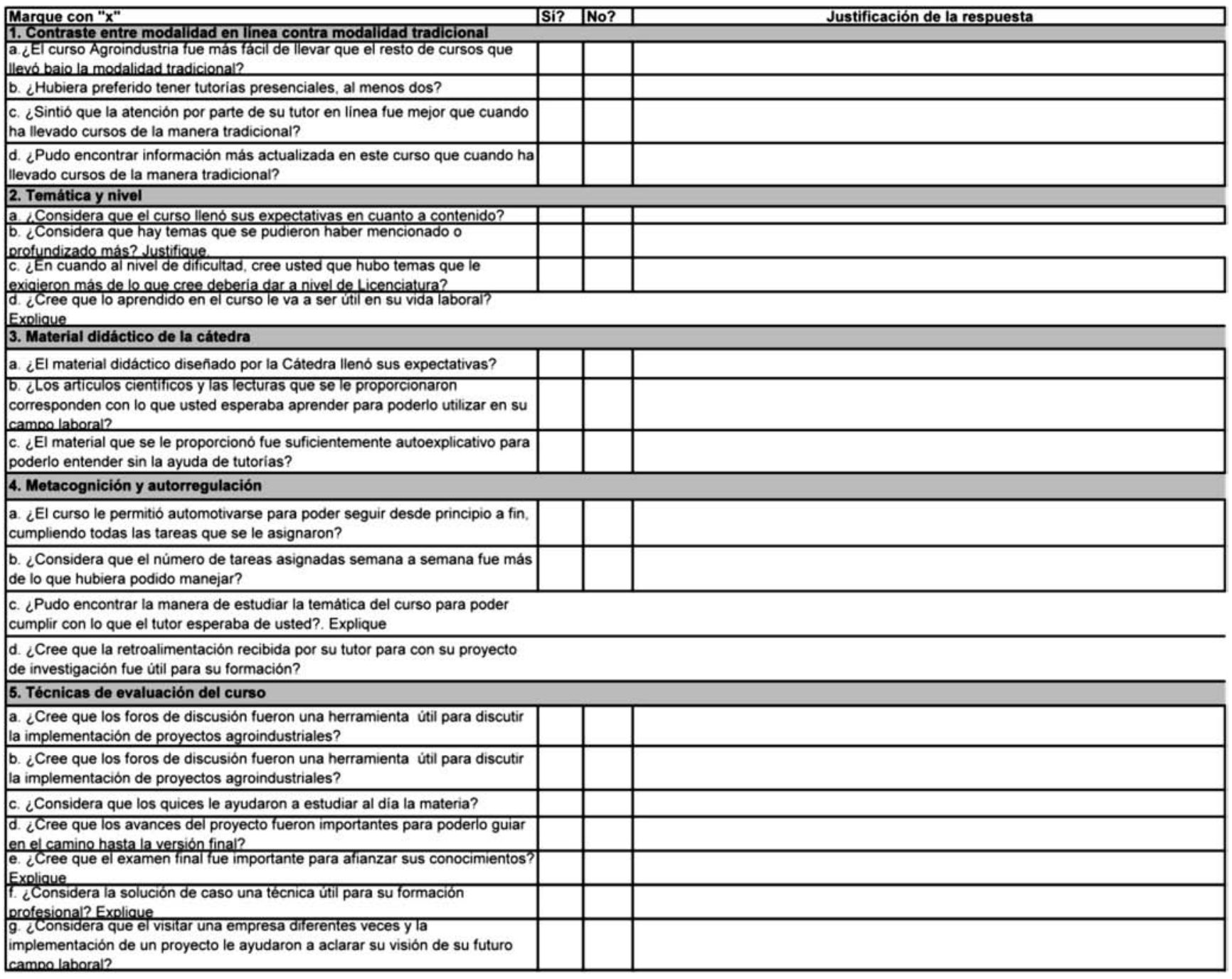

\section{MUCHAS GRACIAS POR SU AYUDA!}




\section{APÉNDICE 7}

Lista de corroboración para evaluar el desempeño del estudiante por parte del tutor durante el desarrollo del Proyecto de Investigación

\section{Lista de corroboración para evaluar el desempeño del estudiante}

Persona que realiza la evaluación:

Fecha:

Aspectos a evaluar en el desempeño del estudiante durante el desarrollo del Proyecto de investigación

\begin{tabular}{|l|l|}
\hline Aspecto & Observación \\
\hline $\begin{array}{l}\text { 1. Selección de la empresa desde un inicio } \\
\text { 2. Detección de las necesidades que puede } \\
\text { resolver }\end{array}$ & \\
\hline $\begin{array}{l}\text { 3. Recolección de información de la empresa } \\
\text { en documento escrito }\end{array}$ & \\
\hline $\begin{array}{l}\text { 4. Corrección de los aspectos en que hace } \\
\text { retroalimentación el tutor }\end{array}$ & \\
\hline $\begin{array}{l}\text { 5. Análisis y reflexión sobre la información } \\
\text { recolectada }\end{array}$ & \\
\hline $\begin{array}{l}\text { 6. Recomendaciones basadas en la } \\
\text { información recolectada }\end{array}$ & \\
\hline $\begin{array}{l}\text { 7. Interés del estudiante por comprender la } \\
\text { retroalimentación del tutor }\end{array}$ & \\
\hline $\begin{array}{l}\text { 8. Desarrollo de la agroindustria rural y } \\
\text { enfoque en ayudar al pequeño empresario }\end{array}$ & \\
\hline
\end{tabular}

\section{APÉNDICE 8}

Resultados obtenidos después de la aplicación de la lista de corroboración para evaluar el desempeño del estudiante durante la elaboración del Proyecto de Investigación del curso de Agroindustria en línea durante el Il cuatrimestre del 2009

\begin{tabular}{|c|c|c|c|c|c|c|c|c|}
\hline & \multicolumn{9}{|c|}{ Número de pregunta } \\
\hline Estudiantes & $\mathbf{1}$ & $\mathbf{2}$ & $\mathbf{3}$ & $\mathbf{4}$ & $\mathbf{5}$ & $\mathbf{6}$ & $\mathbf{7}$ & $\mathbf{8}$ \\
\hline 1 & OK & OK & OK & OK & OK & OK & OK & OK \\
\hline 2 & OK & OK & OK & OK & OK & OK & OK & OK \\
\hline 3 & OK & OK & - & - & - & - & - & OK \\
\hline 4 & OK & OK & - & - & OK & - & - & OK \\
\hline 5 & OK & OK & OK & OK & OK & OK & OK & OK \\
\hline 6 & OK & OK & OK & OK & OK & OK & OK & OK \\
\hline 7 & OK & OK & OK & OK & OK & OK & OK & OK \\
\hline 8 & - & OK & OK & OK & OK & OK & OK & OK \\
\hline 9 & - & OK & - & OK & - & - & & OK \\
\hline 10 & - & - & - & - & - & - & - & OK \\
\hline
\end{tabular}




\section{APÉNDICE 9}

Preguntas utilizadas para calificar el concepto del profesor sobre cada estudiante, de acuerdo a su reacción ante el curso de Agroindustria en línea impartido durante el II cuatrimestre del 2009

1) ¿El alumno tuvo una participación suficiente todas las semanas en todas las actividades realizadas?

2) ¿El estudiante aporta ideas valiosas al tema en cada evaluación que se realizó?

3) ¿El estudiante se interesó por corregir lo que el tutor le indicó como evaluación formativa?

4) ¿El estudiante puede interiorizar aplicando la metacognición, los conocimientos del curso?

5) ¿El estudiante aplica los conocimientos a su futuro campo laboral, ya los estudiantes son de carreras diferentes?

6) ¿El estudiante utiliza bien el medio Web CT para entregar sus trabajos, realizar los quices, participar en los foros, resolver el caso y todas las actividades solicitadas?

7) ¿El estudiante aporta de manera significativa en la zona rural en donde está realizando su trabajo de investigación?

\section{APÉNDICE 10}

Resultados de la aplicación de la escala gráfica para evaluar el concepto de cada estudiante que llevó el curso de Agroindustria en línea durante el II cuatrimestre del 2009

\begin{tabular}{|c|c|c|c|c|c|c|c|}
\hline Alumno & $\mathbf{1}$ & $\mathbf{2}$ & $\mathbf{3}$ & $\mathbf{4}$ & $\mathbf{5}$ & $\mathbf{6}$ & $\mathbf{7}$ \\
\hline 1 & $\mathrm{O}$ & $\mathrm{F}$ & $\mathrm{F}$ & $\mathrm{F}$ & $\mathrm{S}$ & $\mathrm{F}$ & $\mathrm{S}$ \\
\hline 2 & $\mathrm{~S}$ & $\mathrm{~F}$ & $\mathrm{~S}$ & $\mathrm{~S}$ & $\mathrm{~S}$ & $\mathrm{~S}$ & $\mathrm{~S}$ \\
\hline 3 & $\mathrm{O}$ & $\mathrm{O}$ & $\mathrm{O}$ & $\mathrm{F}$ & $\mathrm{F}$ & $\mathrm{F}$ & $\mathrm{S}$ \\
\hline 4 & $\mathrm{~S}$ & $\mathrm{~S}$ & $\mathrm{~S}$ & $\mathrm{~S}$ & $\mathrm{~S}$ & $\mathrm{~S}$ & $\mathrm{~S}$ \\
\hline 5 & $\mathrm{~S}$ & $\mathrm{~S}$ & $\mathrm{~S}$ & $\mathrm{~S}$ & $\mathrm{~S}$ & $\mathrm{~S}$ & $\mathrm{~S}$ \\
\hline 6 & $\mathrm{~F}$ & $\mathrm{O}$ & $\mathrm{O}$ & $\mathrm{S}$ & $\mathrm{F}$ & $\mathrm{S}$ & $\mathrm{S}$ \\
\hline 7 & $\mathrm{RV}$ & $\mathrm{O}$ & $\mathrm{R}$ & $\mathrm{S}$ & $\mathrm{F}$ & $\mathrm{S}$ & $\mathrm{S}$ \\
\hline 8 & $\mathrm{~N}$ & $\mathrm{~N}$ & $\mathrm{~N}$ & $\mathrm{~N}$ & $\mathrm{~N}$ & $\mathrm{~N}$ & $\mathrm{~N}$ \\
\hline 9 & $\mathrm{~F}$ & $\mathrm{~F}$ & $\mathrm{O}$ & $\mathrm{F}$ & $\mathrm{F}$ & $\mathrm{F}$ & $\mathrm{S}$ \\
\hline 10 & $\mathrm{~F}$ & $\mathrm{~F}$ & $\mathrm{O}$ & $\mathrm{F}$ & $\mathrm{F}$ & $\mathrm{F}$ & $\mathrm{S}$ \\
\hline
\end{tabular}

N: Nunca. R: Rara vez. O: Ocasionalmente. F: Frecuentemente S: Siempre 